\title{
Interplay between inflammatory process and platelet activation in chronic spontaneous urticaria
}

\author{
Lu Wang ${ }^{1}$ and Zhiming $\mathrm{Lu}^{2}$ \\ ${ }^{1}$ Qilu Hospital(Qingdao),Cheeloo College of Medicine,Shandong University \\ ${ }^{2}$ Shandong Provincial Hospital
}

June 11, 2020

\begin{abstract}
background. Chronic spontaneous urticaria (CSU) is associated with activation of systemic inflammatory response and platelet activation. To study whether there is a relationship between inflammatory process and platelet activation in CSU patients. Methods. Circulating concentrations of hsCRP, basophil count and mean platelet volume (MPV) were measured in patients with CSU of varying severity as well as in healthy subjects, and we analyzed the relationship between markers and the disease severity and the correlation between markers. Results. The levels of hsCRP and MPV were elevated in patients with CSU,and patients with CSU displayed significantly lower levels of basophil count than the controls. The levels of hsCRP, basophil count and MPV were associated with disease severity. A significant association was found between MPV and hsCRP concentrations in patients with CSU, and basophil count were respectively correlated with MPV and hsCRP. Conclusions. There may be an interplay between inflammatory process and platelet activation in the pathogenesis of CSU.
\end{abstract}

\section{Introduction}

Chronic spontaneous urticaria (CSU) is a multifactorial mast cell-dependent inflammatory disease, which is characterized by recurrent itchy wheals and/or angioedema. Several immunological events have been described in CSU, which are including inflammatory response and platelet activation ${ }^{1-4}$.

It is known, that CRP is produced during an inflammatory reaction and is elevated in various disorders including infections, cancer, autoimmune, cardiovascular and gastrointestinal diseases ${ }^{5-7}$.Recently, hypersensitive C-reactive protein (hsCRP) has been shown to be helpful for potential adjunct for global risk assessment in the primary prevention of cardiovascular disease ${ }^{8}$. Moreover,recent findings demonstrated that basophils may play important roles in a variety of inflammatory skin diseases ${ }^{9}$. On the other hand,the platelet parameters play an impotant and active role in the immune-inflammatory response ${ }^{10,11}$. Mean platelet volume (MPV) is an indicator of platelet size, which has been shown to be the markers of platelet function and activation ${ }^{10}$.

In addition,some studies have reported that the evaluation of disease severity in patients with chronic spontaneous urticaria (CSU) is essential for the adequate treatment of patients, and higher MPV was associated with low-grade inflammatory conditions and a variety of risk factors of cardiovascular disorders ${ }^{10,12}$. The correlation between biomarkers may be important in understanding of pathogenetic processes in CSU and increasing prognostic value ${ }^{2}$, yet the prospective studies focusing on these relationship are still lacking.

Taken together, we sought to see whether there was any correlation between the markers of inflammatory process and platelet activation in CSU. Therefore, we measured circulating concentrations of hsCRP, basophil count and MPV of CSU patients and the healthy subjects, and then analyzed the relationship between markers and the disease severity and the correlation between markers. 


\section{Patients andMethods}

\section{Subjects}

Ninety-eight subjects (males : females $=52: 46 ;$ mean age $=34.5$ years; range $18-70$ years) with CSU were evaluated at Department of Dermatology and selected for the study. The control group consisted of ninty-one healthy subjects, mean age is 36 years ranging from 20 to 60 . All patients fulfilled the EAACI/GA(2)LEN/EDF/WAO criteria for CSU (spontaneous appearance of wheals/no obvious external specific trigger daily, or almost daily for [?] 6 weeks $)^{13}$. All the patients showed active urticaria without angioedema at the time of assessment. None of the examined subjects had taken antihistamines within at least 4 days before the study, glucocorticoids and cyclosporine therapy had been withdrawn at least 8 weeks before.None of these patients were previously treated with omalizumab.Routine investigations had been performed to exclude concomitant diseases/conditions that may lead to an increase in systemic inflammatory response markers, and exclude any known other diseases.

\section{Assessment of disease severity}

According to the mean number of wheals which was appeared during a week, the disease severity was assessed before blood sampling ${ }^{14}$ : patients with 1 to 10 small wheals $(<3 \mathrm{~cm}$ in diameter $)$ were classified as mild, patients with 10 to 50 small wheals or 1 to 10 large wheals ( $>3 \mathrm{~cm}$ in diameter) were classified as moderate,and patients with more than 50 small wheals or less than 10 large wheals were classified as severe.

\section{Blood collection}

For all measurements, venous blood was collected between 8:00 and 10:00 hours from the antecubital vein in the morning after an overnight fast. EDTA was used as anticoagulant. The remaining samples were collected and evaluations were made as part of a standard battery of routine laboratory examinations in CSU patients.

\section{Measurement of whole blood hsCRP,MPV and basophil}

HsCRP concentrations were measured by a immune scattering Nepholometry method using DELTA specific protein immunoanalyzer(Japan).Basophil count and MPV concentrations were measured by Sysmex XN-3000 Hematoiogy System(Japan).All assays were performed according to manufacturer's detailed instructions.

\section{Statistics}

The data in this study were presented as mean \pm standard deviation. Statistical analysis of differences between the two groups was determined by the Unpaired $t$ test. The correlations were assessed by the Spearman's rank test.P $<0.05$ was considered to demonstrate statistical differences. The statistical analysis was performed by graphpad prism (GraphPad Software, San Diego, CA, USA).

\section{Results}

\section{Levels of Whole blood hsCRP , basophil count and MPV in patients with CSU}

Concentrations of hsCRP ,basophil count and MPV in patients with CSU were within their normal ranges. The CSU patients showed significantly higher concentrations of hsCRP and MPV than the controls $(\mathrm{p}<0.0001 ;$ Fig 1 and Table 1$)$. But patients with CSU displayed significantly lower levels of basophil count than the controls $(\mathrm{p}=0.0024$; Fig 1 and Table 1$)$.

\section{Relationship of clinical disease severity towhole blood hsCRP ,basophil count and MPV}

Based on the scale of disease severity, 41 of 98 patients with CSU were evaluated as severe, 57 as moderate and mild. Patients with severe urticaria displayed significantly higher levels of hsCRP and MPV than patients with moderate and mild disease $(\mathrm{p}<0.0001$; Fig 2 and Table 2$)$. Patients with severe urticaria displayed 
significantly lower levels of basophil count than patients with moderate and mild disease $(\mathrm{p}=0.0008$; Fig 2 and Table 2).

\section{Relationship among levels of whole blood hsCRP, basophil count and MPV}

HsCRP were positively correlated with MPV $(r=0.6733, p<0.0001)$, there were significant negatively correlations between basophil count and hsCRP concentrations ( $\mathrm{r}=-0.6720, \mathrm{p}<0.0001 ;$ Fig 3 ), and MPV were negatively correlated with basophil count $(\mathrm{r}=-0.6723, \mathrm{p}<0.0001 ;$ Fig 3$)$.

\section{Discussion}

In this study, significant differences were found in hsCRP, basophil count and MPV between CSU patients and the controls, which supports the assumption that chronic low-grade inflammation and platelet activation are present in $\mathrm{CSU}^{15,16}$. One possible reason for the reduced number of basophils in CSU is that these cells are actively recruited into lesional skin. In the presence of IL-3,chemotaxis is selectively enhanced in basophils by transendothelial migration ${ }^{17}$. The presence of larger platelets in patients might be a consequence of platelets' consumption at the site of the urticarial wheals and further compensatory thrombopoiesis ${ }^{18}$.

Previous studies found a significant association between CSU severity and blood levels of CRP ${ }^{19}$.Ohtsuka T. found that basopenia and high disease activity may be interlinked.Besides a significant positive correlation between CU severity score and MPV in ASST-positive patients was observed by Magen et al. ${ }^{18}$. The present findings are consistent with previous studies.Based on large study it has been indicated that CRP, basophil count and MPV may serve as biomarkers to monitor CSU severity. In addition, We found a positive or negative correlation between these markers, likely reflecting the potential interplay between inflammatory process and platelet activation in CSU.

It is known that CRP increased due to mast cell activation accompanied by inflammatory response ${ }^{20}$. MPV levels correlated with hsCRP concentration, probably reflecting the same characteristic of the inflammatory response in CSU.It suggests that there is an interrelation between the platelets activation and the inflammatory state in the pathogenesis of CSU. Prior studies have consistently demonstrated that basophils play a crucial role in IgE-dependent chronic allergic inflammation in the skin ${ }^{21} \mathrm{John}$ A. Eckman et al. have found altered basophil IgE receptor function in CSU ${ }^{22}$. Moreover,some other studies pointed that platelet activation may occur during immunoglobulin E antibody(1gE)-mediated reactions. Stimulated basophils release preformed histamine, the major mediator of urticaria, and newly synthesized factors, such as leukotriene C4 and interleukin (IL)-4 and IL-13 ${ }^{23}$. Histamine potentiates platelet activity measured by the aggregation induced by different agonists, acting through histamine receptors ${ }^{16}$. Therefore, we suggest that the underlying mechanism for this higher activity of platelets may result from local and/or systemic inflammatory response in CSU.

On the other hand,platelets have been described as a source of inflammatory mediators that are implicated in histamine release from basophil and mast cells ${ }^{16}$. Some studies confirm that platelet-derived supernatants (PDS) induce histamine release from human mixed leukocytes containing basophils, one of the initial target cells in IgE-mediated reactions. Platelets, once activated,can produce a soluble substance or substances which can initiate basophil-mediated reactions, further suggesting that platelet activation can enhance inflammatory reactions ${ }^{24}$. Interestingly, data from experimental studies prove that platelet factor-4 (PF-4) fragment is able to induce rat mast cells to release histamine in a dose-dependent manner.Moreover, it has been shown that histamine is released by human platelets in response to aggregatory and immunological stimuli.

Considering the fact that serum histamine-releasing activity is an important determinant for urticarial severity,it seems that CSU patients should be intensively treated to ensure comprehensive control of their symptoms and to reduce inflammatory process and platelet activation.So it is important to perform repeated blood analyses after symptomatic treatment and CSU remission. Sequential assessments of HsCRP, basophil and MPV concentrations, related to severity of CSU might provide stronger evidence of a causal relationship between platelet activation and inflammation. Therefore, more research is required to prove such hypothesis. The analysis of hsCRP, basophil count and MPV is rapid and inexpensive, therefore combining 
measurement may be a logical and practical tool to improve monitoring of CSU severity in patients with low compliance.

To the best of our knowledge, this study is the first prospective report of the correlation among hsCRP,MPV and basophil count. However, the limitation of the study was that biomarkers concentration all remain within the normal range due to fewer cases. Some improvements may be brought through increasing the number of patients.

In conclusion,markers of inflammatory process (CRP and basophil count) and of platelet activation (MPV) are related to each other in CSU patients, suggesting the possible interaction between these markers in the disease. There may be an interplay between inflammatory process and platelet activation in the pathogenesis of CSU.

The correlation between hsCRP,basophil count and MPV would enable evaluation of the effectiveness of medicines and comparing results of various clinical or epidemiological studies.In addition,further study is needed in order to verify the contribution of biomarkers to the pathological etiology of CSU. Long-term prospective studies are needed to confirm whether this also correlates with disease course.

\section{References}

1. Zuberbier T, Aberer W, Asero R, et al. The EAACI/GA(2) LEN/EDF/WAO Guideline for the definition, classification, diagnosis, and management of urticaria: the 2013 revision and update. Allergy.2014;69(7):868887.

2. Kolkhir P, Andre F, Church MK, Maurer M, Metz M. Potential blood biomarkers in chronic spontaneous urticaria. Clin Exp Allergy.2017;47(1):19-36.

3. Triwongwaranat D, Kulthanan K, Chularojanamontri L, Pinkaew S. Correlation between plasma D-dimer levels and the severity of patients with chronic urticaria. Asia Pac Allergy. 2013;3(2):100-105.

4. Kasperska-Zajac A, Grzanka A, Jarzab J, et al. The association between platelet count and acute phase response in chronic spontaneous urticaria. Biomed Res Int. 2014;2014:650913.

5. Vermeire S, Van Assche G, Rutgeerts P. The role of C-reactive protein as an inflammatory marker in gastrointestinal diseases. Nat Clin Pract Gastroenterol Hepatol. 2005;2(12):580-586.

6. Hage FG. C-reactive protein and hypertension. J Hum Hypertens.2014;28(7):410-415.

7. Kotulska A, Kopec-Medrek M, Grosicka A, Kubicka M, Kucharz EJ. Correlation between erythrocyte sedimentation rate and C-reactive protein level in patients with rheumatic diseases. Reumatologia.2015;53(5):243-246.

8. Ridker PM. High-sensitivity C-reactive protein: potential adjunct for global risk assessment in the primary prevention of cardiovascular disease. Circulation. 2001;103(13):1813-1818.

9. Satoh T, Ito Y, Miyagishi C, Yokozeki H. Basophils infiltrate skin lesions of eosinophilic pustular folliculitis (Ofuji's disease).Acta Derm Venereol. 2011;91(3):371-372.

10. Gasparyan AY, Ayvazyan L, Mikhailidis DP, D.Kitas G. Mean Platelet Volume: A Link Between Thrombosis and Inflammation? Current Pharmaceutical Design. 2011;17(1):47-58.

11. Kasperska-Zajac A, Rogala B. Platelet function in anaphylaxis.J Investig Allergol Clin Immunol. $2006 ; 16(1): 1-4$.

12. Chu SG, Becker RC, Berger PB, et al. Mean platelet volume as a predictor of cardiovascular risk: a systematic review and meta-analysis.J Thromb Haemost. 2010;8(1):148-156.

13. Zuberbier T, Aberer W, Asero R, et al. The EAACI/GA(2)LEN/EDF/WAO guideline for the definition, classification, diagnosis and management of urticaria. Allergy. 2018;73(7):1393-1414. 
14. Sabroe RA, Fiebiger E, Francis DM, et al. Classification of anti-FcepsilonRI and anti-IgE autoantibodies in chronic idiopathic urticaria and correlation with disease severity. J Allergy Clin Immunol. 2002;110(3):492499.

15. Kasperska-Zajac A. Acute-phase response in chronic urticaria.J Eur Acad Dermatol Venereol. 2012;26(6):665-672.

16. Chandrashekar L, Rajappa M, Sundar I, et al. Platelet activation in chronic urticaria and its correlation with disease severity. Platelets. 2014;25(3):162-165.

17. Motoyasu Iikura, Motohiro Ebisawa, Masao Yamaguchi, et al. Transendothelial Migration of Human Basophils. The Journal of Immunology. 2004;173:5189-5195.

18. Magen E, Mishal J, Zeldin Y, et al. Increased mean platelet volume and C-reactive protein levels in patients with chronic urticaria with a positive autologous serum skin test. Am J Med Sci.2010;339(6):504508.

19. Kolkhir P, Altrichter S, Hawro T, Maurer M. C-reactive protein is linked to disease activity, impact, and response to treatment in patients with chronic spontaneous urticaria. Allergy.2018;73(4):940-948.

20. Kasperska-Zajac A, Sztylc J, Machura E, Jop G. Plasma IL-6 concentration correlates with clinical disease activity and serum C-reactive protein concentration in chronic urticaria patients. Clin Exp Allergy. 2011;41(10):1386-1391.

21. Mukai K, Matsuoka K, Taya C, et al. Basophils play a critical role in the development of IgE-mediated chronic allergic inflammation independently of T cells and mast cells. Immunity.2005;23(2):191-202.

22. Eckman JA, Hamilton RG, Gober LM, Sterba PM, Saini SS. Basophil phenotypes in chronic idiopathic urticaria in relation to disease activity and autoantibodies. J Invest Dermatol.2008;128(8):1956-1963.

23. Lourenco FD, Azor MH, Santos JC, et al. Activated status of basophils in chronic urticaria leads to interleukin-3 hyper-responsiveness and enhancement of histamine release induced by anti-IgE stimulus. $\mathrm{Br}$ J Dermatol. 2008;158(5):979-986.

24. Orchard M A, Kagey-Sobotka A, Proud D, M. LL. Basophil histamine release induced by a substance from stimulated human platelets. J Immunol Methods. 1986(136):2240-2244.

\section{Tables}

Table 1. Levels of whole blood hsCRP, basophil count and MPV in CSU patients and healthy controls

\begin{tabular}{lllll}
\hline Patient category & No. of patients & hsCRP $(\mathrm{mg} / \mathrm{L})$ & $\begin{array}{l}\text { Basophil count } \\
\left(10^{9} / \mathrm{L}\right)\end{array}$ & MPV (fL) \\
\hline CSU & 98 & $3.750 \pm 1.610$ & $0.028 \pm 0.012$ & $10.360 \pm 0.776$ \\
control & 91 & $1.465 \pm 1.049$ & $0.033 \pm 0.011$ & $9.513 \pm 0.672$ \\
p value & - & $<0.0001$ & 0.0024 & $<0.0001$ \\
\hline
\end{tabular}

Results are expressed as mean \pm standard deviation.

HsCRP, hypersensitive C-reactive protein ; MPV,mean platelet volume ; CSU, chronic spontaneous urticaria.

Table 2. Levels of whole blood hsCRP, basophil count and MPV in CSU patients with different severity

\begin{tabular}{lllll}
\hline CSU severity & No. of patients & hsCRP $(\mathrm{mg} / \mathrm{L})$ & Basophil count $\left(10^{9} / \mathrm{L}\right)$ & MPV $(\mathrm{fL})$ \\
\hline Mild/Moderate & 57 & $3.200 \pm 1.310$ & $0.031 \pm 0.012$ & $10.080 \pm 0.645$ \\
Severe & 41 & $4.515 \pm 1.690$ & $0.023 \pm 0.010$ & $10.750 \pm 0.780$
\end{tabular}




\begin{tabular}{lllll}
\hline CSU severity & No. of patients & $\mathrm{hsCRP}(\mathrm{mg} / \mathrm{L})$ & Basophil count $\left(10^{9} / \mathrm{L}\right)$ & $\mathrm{MPV}(\mathrm{fL})$ \\
\hline $\mathrm{p}$ value & - & $<0.0001$ & 0.0008 & $<0.0001$ \\
\hline
\end{tabular}

Results are expressed as mean \pm standard deviation.

HsCRP, hypersensitive C-reactive protein ; MPV,mean platelet volume ; CSU, chronic spontaneous urticaria.

\section{Figure legends}

\section{Fig 1}

(a) Levels of hsCRP in CSU patients and healthy controls.HsCRP levels were significantly higher in patients with CSU than in controls

(b) Levels of basophil count in CSU patients and healthy controls.Basophil count levels were significantly lower in patients with CSU than in controls

(c) Levels of MPV in CSU patients and healthy controls. MPV levels were significantly higher in patients with CSU than in controls

\section{Fig 2}

1. Levels of hsCRP in patients with CSU divided into two different subsets according to the severity of the disease (mild/moderate and severe).Patients with severe CSU displayed significantly higher levels of hsCRP than patients with mild/moderate CSU

2. Levels of basophil count in patients with CSU divided into two different subsets according to the severity of the disease (mild/moderate and severe). Patients with severe CSU displayed significantly lower levels of basophil count than patients with mild/moderate CSU

3. Levels of MPV in patients with CSU divided into two different subsets according to the severity of the disease (mild/moderate and severe).Patients with severe CSU displayed significantly higher levels of MPV than patients with mild/moderate CSU

\section{Fig 3}

1. Relationship between levels of hsCRP,MPV in patients with CSU.A significant positively correlation was found between hsCRP and MPV levels

2. Relationship between levels of basophil count and hsCRP in patients with CSU.A significant negatively correlation was found between basophil count and hsCRP levels

3. Relationship between levels of MPV and basophil count in patients with CSU. A significant negatively correlation was found between MPV and basophil count levels

Figures

Fig 1.
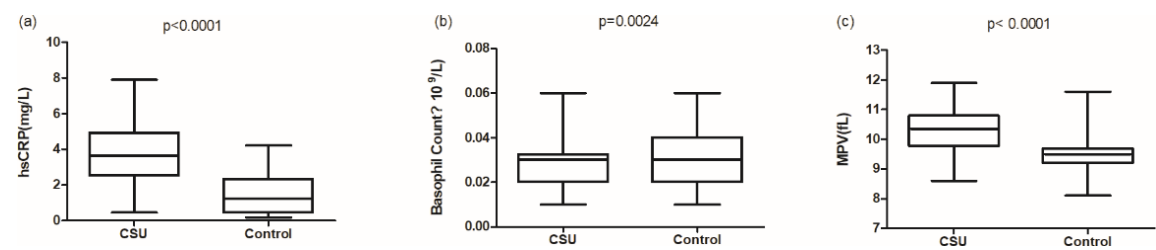

Fig 2. 

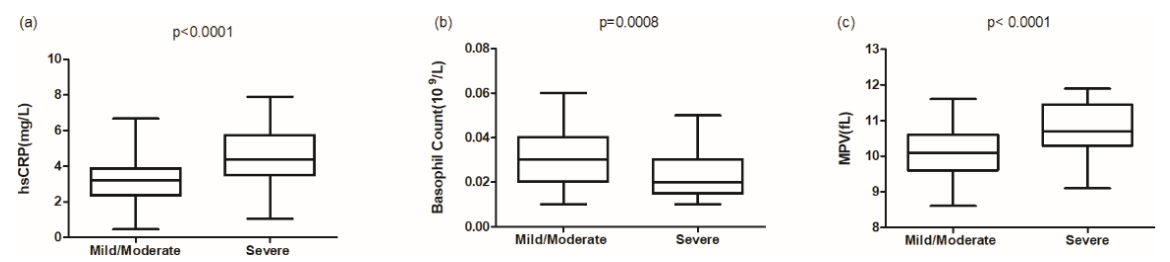

Fig 3.
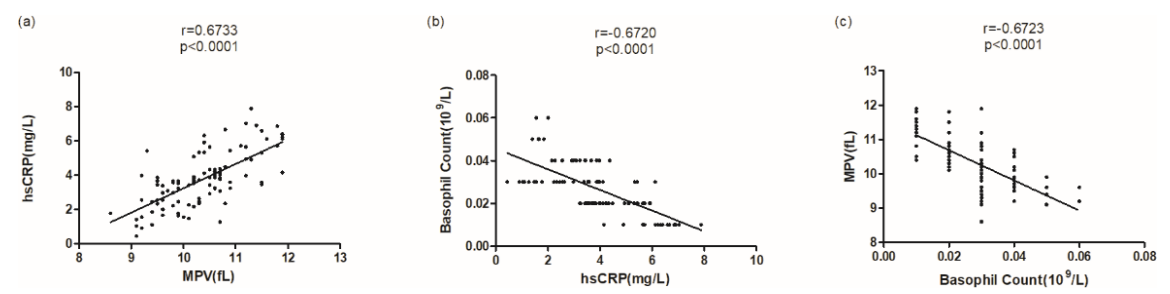